\title{
Contents, Vol. 14, 1905
}

\section{Inhalts - Verzeichnis.}

Opiginalapbeiten.

Bach, L., Traumatische Neurose und Unfallbegutachtung . 246 Bartels, M., Ueber Blutgefässe des Auges bei Glaukom

und übei· experimentelles Glaukom durch Versperrung

von vorderen Blutbahnen. (Hierzu Taf. VII-X) 105, 258, 458 Boldt, J., Kuhnts

Knorpelausschälung in der Trachom-

behandlung 41

Cosmetatos, G. F. , Zur Kenntnis des Kolobom der

Macula lutea 575

Hornicker, E., und Romanin, V., Ueber einen Hülfs-

apparat zur Behandlung des Trachom mit Röntgen-

strahlen $\quad 569$

Ischreyt, G., Zwei Fälle von Xeroderma pigmentosum mit

Tumorbildung an den Lidern 31

Junius, P., Zur Trachonrfrage 452

Jurnitschek, F., Der Innenpol-Magnet. Eine neue Ver-

wertung des Elektromagnetismus zur Entfernung von

Eisensplittern aus dem Auge . • 426, 552

Lohnstein, R., Ueber eine Modifikation des umgekehrten

Bildes und ihre Verwendung zur Refraktionsbestimmung 148 Luerssen, A., Die Beziehungen des

Bazillus Müller zur

Genese des Trachom 443

Matys, v., Die Entwicklung der Tränenableitungswege.

(Hierzu Taf. XV) . $\quad .222$

Michel, J. v., Metastatisehe Aderhaut-Geschwulst bei ver-

mutlicher Hodgkinscher Krankheit ....... 421

Munch, K., Ueber die Innervation der Stromazellen der Iris.

(Hierzu Taf. XI-XII) 130

Oncken, Th., Zur Spätdiagnose traumatischer Netzhaut-

ablösung 165

Schnabel,J., DieEntwicklungsgeschichte der glaukomatösen

Exkavation. (Hierzu Taf. I-VI) 1

Schridde, H., Histologische Untersuchungen der Conjunctivitis gonorrhoica neonatorum. (Hierzu Taf. XVIII) 525 Schultz-Zehden, P., Die chronische, herdförmige Chorio-

Retinitis tuberculosa. (Hierzu Taf. XIII-XIV) . . 213 Stargardt, K., Ueber die Wirkung der Röntgenstrahlen

auf den Trachomfollikel 251 
Stilling, I., Zur Anatomie des myopischen Auges ... 23 Thielemann, R., ZurWirkungsweise der Radiumbestrahlung auf die trachomatöse Bindehaut 559 Vogt, A., Dislocatio lentis spontanea als erbliche Krankheit 153 Wagner, P., Zur Kasuistik der intraokularen Tumoren.

(Hierzu Taf. XVI-XVII) 533

$-\mathrm{IV}-$

Bepieht über die deutsehe ophthalmologisehe Literatur.

Untersuchungsmethoden (I. und II. Semester 1904). Von

Prof. Dr. Dimmer in Graz 55

Mikroorganismen (I. und.1.1. Semester 1903). Von Priv.-Doz.

Dr. Helbron in Berlin ,580

Dioptrik und Anomalien der Refraktion und Akkommodation

(II. Semester 1904 und I.Semester 1905). Von Priv.-Doz.

Dr. Salzmann in Wien59, 590

Anatomie des Auges (II. Semester 1904). Von Prof. Dr.

Sobotta in Würzburg 167

Physiologie des Gesichtssinnes (I. Semester 1904). Von

Priv.-Doz. Dr. Trendelenburg in Freiburg. . . . 295

Berieht über die ausländische ophthalmologisehe Literatur.

Bericht über die amerikanische Literatur (I. Semester 1904).

Von Dr. R. Denig in New York .189

Bericht über die englische ophthalmologisehe Literatur

(II. Semester 1904). Von Dr. R. Gruber in London 307 Bericht über die polnische

ophthalmologisehe Literatur (I.

und II. Semester 1904). Von Dr. v. Kamocki in

Warsehau 313

Bericht über die russische ophthalmologisehe Literatur

(I. und II. Semester 1904). Von Dr. ïh. Werncke

in Odessa und Prof. v. Ewetzky in Dorpat . . 489

Bericht über dies skandinavische Literatur (II. Semester 1904)

193

62

Von Prof. Dr. J. Widmark in Stockholm Bericht über die ungarische Literatur (I. undll. Semester 1904)

Von Prof. Dr. Emil Grósz in Budapest ....

Gesellsehattsberiehte.

Berliner ophthalmologisehe Gesellschaft $\quad 71,511$

Ophthalmologisehe Gesellschaft in Wien ...... 88, 360

St. Petersburger

93

Ungarische - in Budapest $\quad 320$

Ophthalmologisehe Gesellschaft in Heidelberg

Société beige d'ophthalmologie $\quad 610$

française d'ophthalmologie $74,197, \quad 398$

d'ophthalmologie de Paris $\quad 85,405, \quad 614$

Versammlung deutscher Naturforscher und Aerzte in Meran. 
Offene Korrespondenz

Nekrologe: Brettauer

Wilh. Hess

Therapeutisehe ümsehäu

Unfall- und Saehverständigenkunde . . . . 414, 516, 616

T. Tagesnaehriehten 97

210

417, 512

Literatur -Verzeiehnis 100

210

418

$519, \quad 626$

95, 624

417

95, 207, 408, 618 